
allemande

46-2 | 2014

Intellectuels et politique en Allemagne

\title{
Auf der Suche nach der verlorenen Orientierung - Carl Amery: Ein grüner Bewegungsintellektueller zwischen konservativer Bewahrung und progressiver Veränderung
}

Silke Mende

\section{(2) OpenEdition}

\section{Journals}

Édition électronique

URL : https://journals.openedition.org/allemagne/1143

DOI : 10.4000/allemagne. 1143

ISSN : 2605-7913

Éditeur

Société d'études allemandes

Édition imprimée

Date de publication : 30 décembre 2014

Pagination : $365-379$

ISSN : 0035-0974

Référence électronique

Silke Mende, „Auf der Suche nach der verlorenen Orientierung - Carl Amery: Ein grüner Bewegungsintellektueller zwischen konservativer Bewahrung und progressiver Veränderung", Revue d'Allemagne et des pays de langue allemande [Online], 46-2 | 2014, Online erschienen am: 29 Juli 2019 abgerufen am 19 Mai 2021. URL: http://journals.openedition.org/allemagne/1143 ; DOI: https:// doi.org/10.4000/allemagne.1143 


\section{Auf der Suche nach der verlorenen Orientierung - Carl Amery: Ein grüner Bewegungsintellektueller zwischen konservativer Bewahrung und progressiver Veränderung}

- Silke Mende*

Die 1970er und 1980er Jahre des 20. Jahrhunderts wurden bereits aus der Sicht zahlreicher Zeitgenossen als Zeitraum verstärkter Beschleunigung und rasanter Veränderung wahrgenommen ${ }^{(1)}$. Wirtschaftliche Krisen und Strukturbrüche, das Phänomen zunehmender Arbeitslosigkeit, die Präsenz des Ökologiethemas in den öffentlichen Debatten und die so genannte „Krise des Marxismus“ sind nur einige Phänomene, welche für die These eines Umbruchs in der Entwicklung der Moderne ins Feld geführt werden können ${ }^{(2)}$. Im Folgenden rückt ein Thema in den Mittelpunkt, das vielen Zeitgenossen der 1970er und 1980er Jahre als neu und besonders dringlich erschien: die Ökologieproblematik, die seit Beginn der 1970er Jahre massiv auf die öffentliche Agenda drängte und eine Herausforderung für hergebrachte Handlungsmuster und Ordnungsvorstellungen war $^{(3)}$. Wie sollte mit der neu aufscheinenden Problematik verknappter Ressourcen umgegangen werden, die dem Ideal stetig steigenden Wirtschaftswachstums widersprach, das zumal in der bundesdeutschen

* Dr., akademische Rätin auf Zeit am Seminar für Zeitgeschichte der Universität Tübingen.

1 Einige der folgenden Überlegungen wurden bereits in meiner Dissertation ausgeführt. Vgl. dazu: Silke MENDE, „Nicht rechts, nichts links, sondern vorn“. Eine Geschichte der Gründungsgrünen, München, Oldenbourg, 2011, v.a. S. 264-269.

2 Selbstverständlich lassen sich auch Themen benennen, bei denen gegenüber den Brüchen stärker die Kontinuitäten in den Vordergrund treten. Vgl. zu diesen Diskussionen beispielhaft: Konrad H. Jarausch, Das Ende der Zuversicht? Die siebziger Jahre als Geschichte, Göttingen, Vandenhoeck \& Ruprecht, 2008; Anselm Doering-Manteuffel, Lutz Raphael, Nach dem Boom. Perspektiven auf die europäische Zeitgeschichte seit 1970, Göttingen, Vandenhoeck \& Ruprecht, 2012.

3 Vgl. etwa: Kai F. Hünemörder, Die Frühgeschichte der globalen Umweltkrise und die Formierung der deutschen Umweltpolitik (1950-1973), Stuttgart, Steiner, 2004; Joachim RADKAU, Die Ära der Ökologie, München, Beck, 2011; Franz-Josef BRÜGgemeIER, Schranken der Natur. Umwelt und Gesellschaft 1750 2013, Essen, Klartext, 2014. 
Nachkriegsgesellschaft zu einem parteienübergreifenden Konsensprinzip avanciert war? Und welche Bedeutung hatten die in jener Zeit publizierten wissenschaftlichen Prognosen, wie der Bericht an den Club of Rome über die „Grenzen des Wachstums“ (4), sowie die auflagenstarken Katastrophenbestseller mit oftmals apokalyptischer Grundierung, etwa aus der Feder Herbert Gruhls ${ }^{(5)}$, für das Fortschrittsverständnis der modernen Industriegesellschaft?

Die grüne Bewegung, die sich im Laufe der 1970er Jahre im Kontext dieser Diskussionen formierte und 1980 in der Gründung der grünen Bundespartei mündete, versuchte, Antworten auf diese Ungewissheiten zu finden ${ }^{(6)}$. In ihr verdichteten sich die Fragen nach dem Verhältnis von Bewahrung und Veränderung, den Werthaltungen „konservativ“ und „progressiv“ sowie den ideologischen Grundkategorien „rechts“ und „links“. Nicht zufällig sammelten sich die aus zahlreichen unterschiedlichen Richtungen in der grünen Bewegung zusammenströmenden Gruppen, Personen und Programme unter dem Schlagwort: „Wir sind nicht rechts, nicht links, sondern vorn.“

Einer der versiertesten Denker innerhalb der frühen grünen Bewegung war Carl Amery. Der bayerische Schriftsteller, Linkskatholik und überzeugte Konservative lieferte scharfsinnige Beiträge zur Problematik von ökologischer Herausforderung und Fortschrittsverständnis und beeinflusste damit die Debatten in der Öffentlichkeit ebenso wie in seiner eigenen Partei. An seinem Beispiel sollen die Überlegungen der grünen Bewegung zu Moderne und Fortschritt, ökologischer Bewahrung und Veränderung ausschnittartig nachgezeichnet werden. Übergeordnet gerät damit die Frage in den Blick, inwiefern Carl Amery als grüner „Bewegungsintellektueller“ betrachtet werden kann, der über die eigene Partei hinaus - und oft genug im Dissens mit ihr - zu einem Sprachrohr grüner Überlegungen und Ordnungsvorstellungen in der Öffentlichkeit avancierte sowie eine bedeutsame Mittlerperson zu weiteren ökologischen Protagonisten und Vordenkern jenseits der grünen Bewegung wurde. Deshalb gilt es vorab, das Konzept des „Bewegungsintellektuellen“ und seine Bedeutung für die Geschichte sozialer Bewegungen kurz zu umreißen (1), bevor in einem zweiten Teil ein biographischer Abriss zu Carl Amery und seiner Verortung in den Argumentations- und Handlungszusammenhängen seiner Zeit, nicht zuletzt der grünen Bewegung selbst, erfolgt (2). Im Anschluss rückt Amerys Gegenwartsanalyse mit besonderem Augenmerk auf die Kategorie „Fortschritt“ in den Mittelpunkt (3), um schließlich nach seinen Vorschlägen zur Überwindung der von ihm und anderen als krisenhaft empfundenen Situation zu fragen, die in seinem Plädoyer für eine Aufhebung des traditionellen Dualismus von „konservativ“ und „progressiv“ mündeten (4).

4 Dennis Meadows u.a., The limits to growth. A report for the Club of Rome's project on the predicament of mankind, New York, Universe Books, 1972. Zur zeithistorischen Verortung der Studie vgl. u.a. Elke SEEFried, „Towards The Limits to Growth? The Book and its Reception in West Germany and Britain 1972-73“, German Historical Institute Bulletin, 33/1 (2011), S. 3-37.

5 Herbert Gruhl, Ein Planet wird geplündert. Die Schreckensbilanz unserer Politik, Frankfurt am Main, Fischer, 1975.

6 Joachim Raschкe, Die Grünen. Wie sie wurden, was sie sind, Köln, Bund, 1993; S. Mende, „Nichts rechts, nicht links“(Anm. 1). 


\section{1. „Bewegungsintellektuelle“ und Neue Soziale Bewegungen}

Soziale Bewegungen und aus ihr herausgehobene Protagonisten passen auf den ersten Blick nicht recht zusammen. Protestbewegungen tun sich häufig äußerst schwer mit prominenten Vorkämpfern oder gar regelrechten Stars. Umgekehrt fällt es diesen selten leicht, sich mit den oftmals basisdemokratisch grundierten Organisations- und Aushandlungsprinzipien anzufreunden, wie sie vor allem die so genannten Neuen Sozialen Bewegungen seit den späten 1960er Jahren prägten. Das gilt auch für die im letzten Drittel der 1970er Jahre entstehenden Grünen, die sich, zumal in dieser frühen Phase ihrer Geschichte, dezidiert als „Bewegungspartei“ verstanden, welche sich den Wertvorstellungen und Praktiken der Protestbewegungen gegenüber verpflichtet fühlte. In ihren Reihen herrschte stets große Skepsis gegenüber jenen, die ein Parteiamt innehatten, als Abgeordnete im Parlament saßen oder sich gar einer besonderen Beliebtheit bei den Medien, den Wählern oder der Parteibasis erfreuten ${ }^{(7)}$.

Dennoch wurde und wird immer wieder auf die Bedeutung einzelner Protagonisten für soziale Bewegungen hingewiesen. Der Sozialwissenschaftler Joseph Huber, der sich Ende der 1970er Jahre der deutschen Alternativbewegung annahm und selbst in dieser verankert war, sprach von der Bedeutung so genannter „Drehpunktpersonen“. Damit schloss er an Ausführungen Rolf Schwendters an, die dieser 1971 in seiner „Theorie der Subkultur“ gemacht hatte ${ }^{(8)}$. Bei den „Drehpunktpersonen“, den "pivot players“, handele sich um ,jene Personen, die sowohl mit dem Establishment als auch mit einer Subkultur in Interaktion" stünden und somit eine Art Zwischen- oder intermediäre Kultur bildeten, die zwischen den Extremen vermittele ${ }^{(9)}$. Die beiden amerikanischen Bewegungsforscher Ron Eyerman und Andrew Jamison systematisieren ähnliche Überlegungen in ihrer Theorie der sozialen Bewegungen. Deren Formierung deuten sie als einen Prozess, in dem Bedeutung (meaning) und Bewusstsein (consciousness) konstruiert würden. Im Laufe der Zeit verändere sich die solcherart konstruierte kollektive Identität und mit ihr die sie jeweils prägenden Akteurszusammenhänge ${ }^{(10)}$. Für unterschiedliche Phasen und Strömungen innerhalb von sozialen Bewegungen kommen damit spezifische Personengruppen oder einzelne Protagonisten ins Spiel, die prägend sind. Zwar seien, so Eyerman und Jamison, alle Aktiven innerhalb einer sozialen Bewegung an der Bewusstseins- und Bedeutungsbildung, an der Schaffung einer kollektiven Identität beteiligt, und in diesem Sinne als „Intellektuelle“ zu verstehen, aber:

„All activists do not participate equally in the cognitive praxis of social movements, however. Some actors are more visible as organizers, leaders or spokespersons. This visibility,

7 Vgl. Harald Knitter, Basisdemokratie und Medienelite. Die Parteiprominenz der GRÜNEN in der Presse, Münster, LIT, 1998.

8 Joseph Huber, Wer soll das alles ändern? Die Alternativen der Alternativbewegung, Berlin, RotbuchVerlag, 1981, S. 96-99. Neuerdings aus geschichtswissenschaftlicher Perspektive zur Alternativbewegung: Sven ReICHARDT, Authentizität und Gemeinschaft. Linksalternatives Leben in den siebziger und frühen achtziger Jahren, Berlin, Suhrkamp, 2014.

9 Rolf SchwendTER, Theorie der Subkultur. Neuausgabe mit einem Nachwort, sieben Jahre später, Köln, Kiepenheuer \& Witsch, 1978, S. 62.

10 Ron Eyerman, Andrew Jamison, Social Movements. A Cognitive Approach, University Park, PA, Pennsylvania State University Press, 1991. 
often helped along by sources outside the movement like the mass media, is the basis for the usual distinctions between the leaders and the led“(11).

Durch herausragende Persönlichkeiten „sprechen“ soziale Bewegungen als Kollektive. Mittels der näheren Betrachtung ihrer Protagonisten wird ihre oftmals verstreute kollektive Identität greifbar. Diese haben innerhalb einer sozialen Bewegung wiederum unterschiedliche Funktionen. Eine besondere Rolle spielt der Begriff des „Bewegungsintellektuellen“. Anschließend an Antonio Gramsci, der „organische“ von „traditionellen Intellektuellen“ unterscheidet, stellen Eyerman und Jamison den „established intellectuals“ so genannte „movement intellectuals“ gegenüber. Während mit „etablierten Intellektuellen“ diejenigen gemeint sind, die innerhalb traditioneller sozialer und akademischer Institutionen geformt wurden, sind Bewegungsintellektuelle jene, die im Kontext einer sozialen Bewegung zu deren bedeutsamen Stichwortgebern und Leitfiguren werden ${ }^{(12)}$.

Obwohl die Grünen zeitgenössisch wie retrospektiv von sich selbst das Bild einer streng basisbezogenen und egalitären Bewegung zeichnen, erscheint es sinnvoll, einzelne Protagonisten im grünen Entstehungsprozess näher zu betrachten, konzentriert sich in ihrem Denken und Handeln doch brennpunktartig das Selbstverständnis der gesamten Bewegung oder das einzelner Strömungen in einem bestimmten Zeitraum. Grüne Bewegungsintellektuelle wie etwa der in diesem Beitrag im Mittelpunkt stehende Carl Amery bereiteten durch ihre Ideen und Überlegungen, durch ihre Netzwerke und Kontakte das Feld der Formierung. Zudem verliehen sie unterschiedlichen dabei verhandelten Positionen und Strömungen Ausdruck. In Bewegungsintellektuellen verdichteten sich bestimmte Überlegungen und Konzepte, die innerhalb der grünen Bewegung als ganzer oder innerhalb ihrer einzelnen Strömungen diskutiert wurden.

\section{Carl Amery als grüner Bewegungsintellektueller}

Carl Amery wurde 1922 in München als Christian Anton Mayer geboren und wuchs in Passau und Freising auf ${ }^{(13)}$. Bayern sollte zeit seines Lebens lebensweltlicher Ankerund Bezugspunkt bleiben. Über sein Herkunftsmilieu, das ihn politisch und ideologisch stark geprägt hat, schreibt Amery 1983:

„Das konservative Milieu, in dem ich aufwuchs, war das des süddeutschen Bildungskatholizismus; also ein Konservativismus mit stark, alt-großdeutschen' Ober- und Untertönen. Mit der Romantik teilte er eine grundsätzlich mißtrauische Einstellung gegenüber dem historischen Erfolg, der keineswegs als Kriterium historischer ,Richtigkeit' empfunden

11 Ebd., S. 94.

12 Ebd., S. 94-97.

13 Hierzu und zum Folgenden: Carl Amery, Lebenslauf. Anlage zu Amery, Carl, Brief an Heinrich Häberlein, Deutsche Friedensgesellschaft vom 3. Mai 1984 (Monacensia. Literaturarchiv und Bibliothek München [fortan: Monacensia], Nachlass: Carl Amery, Mappe: Briefe an Friedensgesellschaft Dt.); Carl Amery, Ist ökologischer Humanismus möglich?, Rede gehalten auf dem Seminar der E.-F.-Schumacher-Gesellschaft „Ist ökologischer Humanismus möglich?“ am 14./15.2.1981 (Petra-Kelly-Archiv im Archiv Grünes Gedächtnis [fortan: PKA], Nr.: 2474 [1]); vgl. außerdem: Joseph Kiermeier-Debre, Carl Amery. „...ahnen, wie das alles gemeint war“. Ausstellung eines Werkes, München/Leipzig, List, 1996. 
wurde. Kritik am Industriesystem und seinen politischen Organisationsformen war in diesem Milieu gang und gäbe“(14).

Der Kernbestand von Amerys Wertvorstellungen, darüber hinaus spezifische Kritikmuster, die in den 1970er und 1980er Jahren handlungsleitend werden sollten, schienen hier bereits angelegt.

Nach Krieg und amerikanischer Gefangenschaft orientierte sich Amery beruflich mit einem Studium der Neuphilologie in München und Washington schnell in die literarische Richtung ${ }^{(15)}$. 1954, bereits unter seinem Pseudonym, erschien sein erster Roman, in dessen Folge er regelmäßiger Gast bei den Treffen der Gruppe 47 wurde. Weitere Romane und Veröffentlichungen folgten, darunter 1963 die Streitschrift Die Kapitulation $^{(16)}$, in der sich der Autor mit seinem katholischen Herkunftsmilieu und dessen Rolle im „Dritten Reich“ auseinandersetzt und die ihm wohl auch das Etikett „linkskatholisch“ eingetragen hat. In der Folge gewann der Schriftsteller innerhalb des bundesdeutschen literarischen Betriebs an Renommee: Von 1967 bis 1971 war er Leiter der Städtischen Bibliotheken in München, 1976/77 Vorsitzender des Verbandes der deutschen Schriftsteller, von 1989 bis 1991 Präsident des deutschen PENZentrums. Mitte der 1970er Jahre erfolgte in seinem Werk eine Hinwendung zum Science-Fiction-Genre, die vor dem Hintergrund der zunehmenden Aufmerksamkeit des Schriftstellers für Ökologiefragen sowie Fortschritts- und Zukunftsbelange als durchaus schlüssige Schwerpunktverlagerung erscheint.

Neben seiner literarischen Tätigkeit war Amery seit der Nachkriegszeit stets politisch aktiv. Sein Engagement brachte wechselnde parteipolitische Zugehörigkeiten mit sich, wies inhaltlich jedoch dauerhafte Konstanten auf ${ }^{(17)}$. Die beherrschenden Themen seiner politischen Biographie waren Frieden und Umwelt. 1952 hatte sich Amery an der „Notgemeinschaft gegen Wiederaufrüstung“ beteiligt und war Mitglied der Heinemannschen „Gesamtdeutschen Volkspartei“ geworden. Er engagierte sich im Rahmen der Aktion „Kampf dem Atomtod“, wurde Gründungsmitglied des „Komitees gegen Atomrüstung“ und nahm an der Ostermarschbewegung teil. 1967 trat Amery in die SPD ein. In Wählerinitiativen und auf Wahlkampfveranstaltungen versuchte er früh, wenn auch weitgehend vergeblich, das Ökologie-Thema bei den Sozialdemokraten zu platzieren ${ }^{(18)} .1974$ verließ er die Partei resigniert. Den Ausschlag hatte, wie er in einem in seinem Nachlass dokumentierten Lebenslauf schreibt, seine „intensive Beschäftigung mit der ökologischen Frage “(19) gegeben. Der Kanzlerwechsel

14 Carl Amery, „Deutscher Konservatismus und der faschistische Graben. Versuch einer zeitgeschichtlichen Bilanz", in: Wolf Schäfer (Hg.), Neue Soziale Bewegungen. Konservativer Aufbruch im bunten Gewand? Arbeitspapiere einer Diskussionsrunde, Frankfurt am Main, Fischer, 1983, S. 11-19, hier: S. 11f.

15 Hierzu und zum Folgenden: J. Kiermeier-Debre, Carl Amery (Anm. 13); Stephen Smith, Michael Töteberg, „Carl Amery“, in: Heinz Ludwig Arnold (Hg.), Kritisches Lexikon zur deutschsprachigen Gegenwartsliteratur, München, Ed. Text \& Kritik, 1999 (31. Nachlieferung).

16 Carl Amery, Die Kapitulation oder Deutscher Katholizismus heute, Reinbek bei Hamburg, Rowohlt, 1963.

17 Hierzu und zum Folgenden: C. Amery, Lebenslauf(Anm. 13).

18 Vgl. z.B. Carl Amery, Alarm im Raumschiff. Vortrag 1970; Wähler-Init[iative] SPD für die bayerische Landtagswahl (Monacensia, NL Carl Amery, Manuskripte: CA M 517 [Ms. 517]).

19 C. Amery, Lebenslauf(Anm. 13). 
von Brandt zu Schmidt symbolisierte für ihn die Wende der SPD hin zu einer auf Nüchternheit, Machbarkeit und auf bloße wirtschaftliche Erfolgszahlen konzentrierten Partei ${ }^{(20)}$. Mit seinem SPD-Austritt ging Amery den Weg zahlreicher bekannter Gründungsgrüner, welche die Sozialdemokraten im Laufe der 1970er Jahre verlassen hatten. Das bekannteste Beispiel ist sicherlich Petra Kelly ${ }^{(21)}$. Mit jenen kritischen Denkern, die sich der Ökologieproblematik annahmen und in der SPD verblieben waren, blieb Amery trotzdem in engem Kontakt. Dazu zählten insbesondere Freimut Duve und Erhard Eppler, letzterer galt als prominentester ökologischer Vordenker innerhalb der SPD. Hinzu kamen Kontakte zu den Kreisen undogmatischer Sozialisten, der außerhalb der SPD organisierten, nicht Moskau-treuen Linken ${ }^{(22)}$. Im Zuge seines Engagements für die ökologische Sache baute Amery darüber hinaus Brücken zur klassischen Naturschutzbewegung und kritischen Wissenschaftlern, wie Klaus Traube, Frederic Vester, Ivan Illich und Manfred Siebker ${ }^{(23)}$.

An der Formierung der grünen Bewegung auf deutscher und insbesondere auf westeuropäischer Ebene seit Mitte der 1970er Jahre war Amery entscheidend beteiligt. Ende 1976 gründete er mit westeuropäischen Mitstreitern die Diskussionsplattform Ecoropa, die für die Entstehung einer grenzüberschreitenden grünen Bewegung in Europa eine bedeutsame Rolle spielte. Es handelte sich um einen losen Zusammenschluss von Umweltaktivisten aus 14 Ländern, die neben öffentlichem Widerstand gegen die ökologische Bedrohung vor allem Theoriearbeit leisten wollten ${ }^{(24)}$. Die Idee, mit einer eigenen grünen Liste zu den ersten europäischen Parlamentswahlen 1979 anzutreten, nimmt in den Überlegungen der Gruppe erstaunlich früh Gestalt an. Im Juni 1976 schreibt Amery an den Vorsitzenden des Bund Naturschutz Bayern, Hubert Weinzierl:

„Ich hatte dort [in Frankreich] [...] eine längere Konferenz mit meinem französischen Herausgeber und anderen prominenten Kämpfern der ökologischen Bewegung. Die allgemeine Ansicht war, daß wir aus unseren jeweiligen regionalen Schneckenhäusern heraus müssen, daß es nicht um ein Europa der Vaterländer, sondern der Heimaträume geht. Ja, man überlegt sich schon, ob nicht möglicherweise bevorstehende europäische Parlamentswahlen der ideale Anlaß sein könnten, ein solches Europa auf einer unabhängigen Liste der Öffentlichkeit anzubieten“ ${ }^{\text {(25). }}$.

20 Carl Amery an die Sozialdemokratische Partei Deutschlands, 7. Dezember 1974 (Monacensia, NL Carl Amery, Mappe: Briefe an [die] SPD); Carl Amery, „Rachel, Lea \& der deutsche Schweinehund“, in: Carl Amery, Bileams Esel. Konservative Aufsätze, München, List, 1991, S. 50-57.

21 Vgl. zu Petra Kelly: Saskia Richter, Die Aktivistin. Das Leben der Petra Kelly, München, DVA, 2010.

22 Z.B.: Carl Amery an Georg Habich, c/o Sozialistisches Büro, Hamburg, 25. August 1978 (Monacensia, NL Carl Amery).

23 Vgl. jeweils die entsprechenden Briefwechsel und Hinweise im Nachlass (Monacensia, NL Carl Amery).

$24 \mathrm{Zu}$ Ecoropa gehörten zeitweilig etwa der konservative britische Ökologe Edward Goldsmith, außerdem die Futurologen Ossip K. Flechtheim und Robert Jungk, schließlich Denis de Rougemont und Aurelio Peccei. Prominente Unterstützer waren beispielsweise das österreichische Ökologenpaar Paul Blau und Freda Meissner-Blau, die französischen Grünen Brice Lalonde und Solange Fernex. Vgl.: Pour une démocratie écologique (sans date) (PKA, Nr.: 953); Positionspapier Ecoropa, Oktober 1977 (PKA, Nr.: 2993).

25 Carl Amery an den Bund Naturschutz in Bayern e.V., z. Hd. Herrn [Hubert] Weinzierl, 1.6.1976 (Monacensia, NL Carl Amery, Mappe: Verlage an Amery: Ökologie). 
Als sich in der Bundesrepublik seit 1977 grüne Listen gründeten und zu Wahlen antraten, wurde dies von Amery mit großer Aufmerksamkeit verfolgt ${ }^{(26)}$. Er beteiligte sich an der Formierung der bayerischen Grünen, wurde Gründungsmitglied der Bundespartei und war Mit-Organisator einer prominenten Wählerinitiative für den Europawahlkampf der Partei 1979, an der unter anderem Heinrich Böll, Ossip K. Flechtheim und Helmut Gollwitzer beteiligt waren ${ }^{(27)}$. Im Gründungsjahr der Bundespartei, 1980, rief er die parteinahe „Ernst-Friedrich-Schumacher-Gesellschaft für politische Ökologie“ ins Leben, die sich ebenfalls auf die Bearbeitung theoretischer Fragestellungen konzentrierte ${ }^{(28)}$. Durch dieses Engagement vor, während und nach der Gründung der Partei wurde Amery zu einem regelrechten Bewegungsintellektuellen der Grünen ${ }^{(29)}$. Eine oftmals forsch vorgetragene Theorieabstinenz als genuines Identitätsmerkmal weiter Teile der Umwelt- und Friedensbewegung begriff er als strukturelles Problem, das es zu beheben gelte:

„Wahllos alle möglichen Schriftsteller anzuschreiben, ob sie nicht einen Beitrag zur Anthologie ,Meine Marienwallfahrt' liefern könnten, oder den Dalai Lama zusammen mit HopiIndianern einer Presseöffentlichkeit der Frankfurter Buchmesse zu präsentieren, scheint mir nicht ganz der richtige Weg zu sein“(30).

Hier lagen auch die Sollbruchstellen zwischen Amery und den Grünen: Da der Basisbezug geradezu als ein spezifischer grüner Erinnerungsort gepflegt wurde und die Partei sich stets mit herausgehobenen Persönlichkeiten oder gar intellektuellen Vordenkern schwertat, kam es nicht selten zu Verstimmungen zwischen Amery und seiner Partei. In deren Folge erklärte er 1988 seinen Parteiaustritt ${ }^{(31)}$. Die Grünen waren in seinen Augen „zu einem preisgünstigen Angebot auf den Wühltischen der Betroffenheitsindustrie, der Esoterik, des New-Age-Rummels “ ${ }^{\text {(32) }}$ geworden. Trotzdem begleitete er die grüne Sache weiterhin mit Interesse und Sympathie. Im Mai 2005 starb Carl Amery in München.

\section{Fortschritt im Zeitalter der ökologischen Bedrohung}

Seit den späten 1960er Jahren wurde das Umweltthema zum Schwerpunkt in Amerys politischer und publizistischer Arbeit. Die „ökologische Rettung“, so Amery, sei das "gesellschaftliche, wirtschaftliche, politische Problem Nummer eins der Epoche “(33).

26 C. Amery, Lebenslauf(Anm. 13).

27 Ossip K. Flechtнeim, Wählerinitiative für „Die Grünen“ und ihre Forderungen zu den Europawahlen am 10. Juni 1979 (Archiv Grünes Gedächtnis [fortan: AGG]: B. I. 1. - BuVo/BGSt 1979-1989, Nr. 224); Carl Amery an Helmut Gollwitzer, 30. April 1979 (Monacensia, NL Carl Amery, Mappe: Briefe an Helmut Gollwitzer).

28 Zu Organisation und Programmatik: Konzeptpapier zur E.-F.-Schumacher-Gesellschaft (PKA, Nr.: 985).

29 Vgl. Abschnitt 1 dieses Beitrags.

30 C. Amery, „Deutscher Konservatismus“ (Anm. 14), S. 19.

31 Carl Amery an Die Grünen KV München-Ost, 19. Mai 1988 (Monacensia, NL Carl Amery, Mappe: Briefe an Die Grünen).

32 C. Amery zit. in: S. Smith, M. Töteberg, „Carl Amery“ (Anm. 15), S. 10.

33 Carl Amery, „Lebensqualität - Leerformel oder konkrete Utopie?“, in: Uwe Schultz (Hg.), Lebensqualität. Konkrete Vorschläge zu einem abstrakten Begriff, Frankfurt am Main, Aspekte-Verlag, 1975, S. 8-20, hier: S. 16. 
Bis vor kurzem, so schrieb er 1976, sei die Drohung einer totalen Vernichtung der Erde noch ausschließlich von „der Kriegsmaschinerie, nämlich der Atombombe“ ausgegangen. In den vergangenen Jahren hätten sich nun jedoch die Schwerpunkte verschoben: Es sei „die handfeste Friedenspraxis der Expansion, vor allem der wirtschaftlichen, die uns sozusagen nötigt, das Floß aufzufressen, auf dem wir schwimmen“ ${ }^{(34)}$. Die Verschränkung militärischer mit ökologischen Bedrohungsmomenten stellte für Amery die bedeutendste Frage seiner Zeit dar. Bereits der erste erfolgreiche Atombombenversuch der Amerikaner 1945 in Los Alamos hatte die Möglichkeit einer vollständigen Vernichtung der Menschheit in die Aufmerksamkeit der Weltöffentlichkeit gerückt ${ }^{(35)}$. In den 1970er und frühen 1980er Jahren, in denen zunächst das Umwelt-, dann das Friedensthema erneut prominent diskutiert wurden, rückte diese Perspektive wieder stärker in das Wahrnehmungsfeld der Zeitgenossen. Das Engagement Hunderttausender in Umwelt-, Anti-AKW- und Friedensbewegung, überall in Westeuropa, war beredter Ausdruck dieser Ängste ${ }^{(36)}$.

Ihre Sprengkraft bezogen diese Diskussionen auf einer übergeordneten Ebene durch eine damit verbundene implizite Herausforderung des Fortschrittsdenkens der Moderne, wie es sich seit der Aufklärung entwickelt hatte ${ }^{(37)}$. Nicht nur das Fortschrittsverständnis der liberalen Moderne - in dieser Definition als stetiges lineares Fortschreiten hin zum Besseren begriffen - schien infrage gestellt, sondern bereits der bloße Prozess des Fortschreitens in die Zukunft, die Existenz von Menschheit und Planet. Die Bedrohung gewann in der Wahrnehmung Amerys und anderer Zeitgenossen eine neue drängende Qualität. Die Wurzel des Fortschrittsverständnisses der Moderne, das den Menschen als handelndes Subjekt der Geschichte und Motor des Fortschritts ins Zentrum stellte ${ }^{(38)}$, sah der gläubige Katholik Amery bereits im jüdisch-christlichen Weltbild angelegt ${ }^{(39)}$. Die biblische Formel „Macht Euch die Erde untertan“ (Genesis, 1, 28) habe dank einer kapitalen Fehlinterpretation über Jahrhunderte hinweg ein verhängnisvolles Fortschrittsverständnis begründet, das den

34 Carl Amery, Progressismus, Konservatismus - Positionen in der ökologischen Krise (1976) (Monacensia, NL Carl Amery, Manuskripte: Ms. 358 [CA M 358]), S. 1.

35 Spencer A. WeArt, Nuclear Fear. A History of Images, Harvard/Cambridge/London, Harvard University Press, 1988; Holger Nehring, „Cold War, Apocalypse and Peaceful Atoms. Interpretations of Nuclear Energy in the British and West German Anti-Nuclear Weapons Movements", Historical Social Research, 29/3 (2004), S. 150-170.

36 Lawrence S. Witt tner, Toward Nuclear Abolition. A History of the World Nuclear Disarmament Movement, 1971 to the Present, Stanford, CA, Stanford University Press, 2003; Christoph BeCKer-SCHAUM u.a. (Hg.), „Entrüstet Euch!“ Nuklearkrise, NATO-Doppelbeschluss und Friedensbewegung, Paderborn u.a., Schöningh, 2012.

37 Zu diesem Fortschrittsverständnis vgl.: Reinhart KosElleck, „Fortschritt“, in: Otto Brunner, Werner Conze, Reinhart Koselleck (Hg.), Geschichtliche Grundbegriffe. Historisches Lexikon zur politisch-sozialen Sprache in Deutschland, Bd. 2: E - G, Stuttgart, Klett, 1975, S. 351-423, hier: S. 351-353 u. S. 371-423; Johannes Rонвеск, Die Fortschrittstheorie der Aufklärung. Französische und englische Geschichtsphilosophie in der 2. Hälfte des 18. Jahrhunderts, Frankfurt am Main, Campus, 1987.

38 R. Koselleck, „Fortschritt“ v.a. (Anm. 37), S. 352 u. 397f. Differenzierend: J. Rohвeck, Fortschrittstheorie (Anm. 37), S. 241-247.

39 Ausführlich: Carl Amery, Das Ende der Vorsehung. Die gnadenlosen Folgen des Christentums, Reinbek bei Hamburg, Rowohlt, 1972. 
Menschen in den Mittelpunkt rücke und die natürlichen Grundlagen der Schöpfung seinem autonomen Machtanspruch unterwerfe ${ }^{(40)}$. Diese Tendenz sei nochmals verstärkt worden durch den „völlig missverstandene[n] Kulturauftrag“ der Neuzeit, der Naturbeherrschung als zentrales Anliegen formuliere und mithilfe des technischen Fortschritts auch zunehmend realisiert habe ${ }^{(41)}$.

In einer Besprechung zu Ernst Blochs Prinzip Hoffnung fragte sich Amery deshalb, „ob wir angesichts der höchst realen Drohung unseres planetarischen Daseins noch an der raum- und zeitfreien Definition des MENSCHEN als einer absoluten Größe an sich festhalten wollen und können “(42). Das im Kern anthropozentrische Weltbild der Neuzeit gelte es ad acta zu legen ${ }^{(43)}$ und stattdessen den Menschen als ein in komplexe natürliche Kreisläufe eingebundenes Wesen zu begreifen. Dieses Plädoyer wurde begleitet von einer Argumentationsfigur, die nicht nur in der Wissenschaft Ökologie, sondern auch in den öffentlichen ökologischen Diskussionen der 1970er Jahre eine zunehmend wichtige Rolle spielte: das Denken in netzwerkartigen Beziehungen und Kreislaufbewegungen ${ }^{(44)}$. Es verrät den Einfluss der Arbeiten von kybernetisch arbeitenden Naturwissenschaftlern, allen voran Frederic Vester ${ }^{(45)}$. Die Idee, vorderhand materiellen Fortschritt auf der Grundlage unbegrenzter wirtschaftlicher Wachstumshoffnungen zu erzielen, bezeichnete Amery dementsprechend als die „Folge eines linearen, letzten Endes primitiven Denkens“ (46). Stattdessen operiere das Leben „dezentral, das heißt in Netzen von vielfältigen Beziehungen ohne hierarchischen Mittelpunkt“, während der westliche Zivilisationsentwurf immer stärker $\mathrm{zu}$ „Zentralisierung und Vereinheitlichung“ hindränge ${ }^{(47)}$.

Amerys Kritik an einem anthropozentrischen und linear konzipierten Fortschrittsbegriff bedeutete jedoch nicht, dass er Fortschritt als sinnstiftendes Moment der Gesellschaften im späten 20. Jahrhundert prinzipiell ablehnte. Damit unterschied

40 C. Amery, Progressismus, Konservatismus (Anm. 34), S. 4.

41 Carl Amery, „Der missverstandene Kulturauftrag - Müssen wir die Natur besiegen?“, in: Hermann Benz (Hg.), Grüne zum Kirchentag 1981, Villingen-Schwenningen, 1981, S. 17f.

42 Carl Amery, „Die letzte Prophetie. Carl Amery über, Das Prinzip Hoffnung von Ernst Bloch“, Die Welt, 2.8.1973 (abgedruckt in: J. Kiermeier-Debre, Carl Amery [Anm. 13], S. 95-100, hier: S. 99 [Hervorhebung im Original]).

43 Carl Amery, Natur als Politik. Die ökologische Chance des Menschen, Reinbek bei Hamburg, Rowohlt, 1976, S. 119.

44 Alexander Schmidt-Gernig, „Das Jahrzehnt der Zukunft - Leitbilder und Visionen der Zukunftsforschung in den 60er Jahren in Westeuropa und den USA“, in: Uta GERHARDT (Hg.), Zeitperspektiven. Studien zur Kultur und Gesellschaft. Beiträge aus der Geschichte, Soziologie, Philosophie und Literaturwissenschaft, Stuttgart, Steiner, 2003, S. 305-345; Pascal EiTlER, „Körper - Kosmos - Kybernetik. Transformationen der Religion im ,New Age` (Westdeutschland 1970-1990)“, Zeithistorische Forschungen/Studies in Contemporary History, 4/1-2 (2007), S. 116-136.

45 Der Biochemiker und Umweltexperte Frederic Vester (1925-2003) prägte den Begriff des ,vernetzten Denkens“. Vgl. z.B. Frederic Vester, Das kybernetische Zeitalter. Neue Dimensionen des Denkens, Frankfurt am Main, Fischer, 1974; Frederic VESTER, Unsere Welt-ein vernetztes System. Eine internationale Wanderausstellung, Stuttgart, Klett-Cotta, 1978.

46 Carl Amery, Neue Dimensionen der Verantwortung. Zur Frage der Wachstumsgrenzen (1975), S. 6 (Monacensia: NL Carl Amery, Sign.: CA M 109, Ms. 109).

47 Carl Amery, Neuer Grundwert Lebensqualität - Umweltschutz (Monacensia, NL Carl Amery, Manuskripte: CA M 521 [Ms. 521], S. 2f.), (Hervorhebungen im Original). 
er sich deutlich von anderen ökokonservativen Mahnern. In den Reihen der Gründungsgrünen distanzierte er sich vor allem von Herbert Gruhl, der 1975 mit seinem Katastrophenbestseller Ein Planet wird geplündert große Bekanntheit erlangt hatte ${ }^{(48)}$. Anders als Amery betrachtete der ehemalige CDU-Umweltexperte das Fortschrittsdenken der Aufklärung als Grundübel der Umwelt- und Menschheitskrise in den 1970er Jahren und empfahl die Rückkehr zu vor-aufklärerischen Prinzipien als geistiges, ein strenges Austeritätsprinzip als wirtschaftliches Regenerationsprogramm. Diese Vorstellungen wies Amery entschieden zurück und warnte vehement vor einem Konservativismus, der Stillstand und Umkehr predige ${ }^{(49)}$. Der Sprung des Erkenntnisvermögens, den griechische Philosophie, Altes und Neues Testament, Renaissance und Humanismus bezeugten, sei nicht widerrufbar ${ }^{(50)}$. Stattdessen sollte Fortschritt im Zeitalter der ökologischen Bedrohung neu definiert werden, eine Rückbesinnung auf den „wahren Fortschritt“ und dessen humanistische Grundlagen sei erforderlich. In einem Beitrag aus dem Jahr 1978 äußerte sich Amery dazu folgendermaßen:

„Nichts hat der Idee des Fortschritts mehr geschadet als das Durcheinander, welches das Wort heute noch in den Köpfen erzeugt. [...] In Wahrheit müssen wir, wenn wir mit der Krise fertigwerden wollen, zwei Dinge auseinanderhalten, die man gewöhnlich durcheinanderschmeißt: nämlich den Fortschritt des menschlichen Bewusstseins, der wachsenden Einsicht in das, was uns zur Bewältigung unseres Lebens nottut, und den Fortschritt als reine Vermehrung von Zeugs, so wie sich ein Affe Lianen oder Schmuckketten umhängen mag in der vagen Annahme, daß er dadurch an existenzieller Macht gewinne“(51).

Dennoch:

„Fortschritt ist allemal eine Erweiterung des Bewusstseins, die Ahnung neuer Mündigkeit - aber auch damit verbundener neuer Verantwortlichkeit. [...] Er [der einzige wahre Fortschritt] hat die Modellfigur einer Spirale, die - scheinbar - auf Umwegen von unten nach oben läuft; sie durcheilt dabei immer wieder Bewusstseinsfelder, Traditionen, die in der Geschichte schon einmal vorlagen“ “(52).

Der „wahre“ Fortschritt sei also das Fortschreiten des menschlichen Bewusstseins. Amery wandte sich dementsprechend scharf gegen eine Reduzierung von Fortschritt auf Wirtschaftswachstum. Ein solchermaßen lediglich materiell begründeter Fortschrittsbegriff präge weite Teile der westlichen Marktwirtschaften ebenso wie der östlichen Planwirtschaften.

Mit diesen Überlegungen formuliert Amery Vorstellungen, die bei den frühen Grünen weit verbreitet waren. Deren Denken war häufig von einem intensiven Krisendiskurs gezeichnet, der manchmal in geradezu apokalyptischen Vorstellungen gipfelte ${ }^{(53)}$.

48 H. Gruhl, Planet (Anm. 5).

49 Carl Amery, „Grüne Wähler - Rote Theorie?“, in: Rudolf Brun (Hg.), Der grüne Protest. Herausforderung durch die Umweltparteien, Frankfurt am Main, Fischer, 1978, S. 81-91, hier: S. 86f.

50 C. Amery, Natur als Politik (Anm. 43), S. 37.

51 Carl Amery, Warum brauchen wir die Grünen. Anläßlich der bayerischen Landtagswahl 1978 gehaltener Vortrag, Hamburg, 1978, S. 8 .

52 C. Amery, Natur als Politik (Anm. 43), S. 178-180.

53 Annekatrin Gebauer, „Apokalyptik und Eschatologie. Zum Politikverständnis der GRÜNEN in ihrer Gründungsphase“, Archiv für Sozialgeschichte, 47 (2007), S. 47-93; S. MENDE, „Nicht rechts, nicht links“(Anm. 1), S. 365-406. 
Im Zuge der in den 1970er Jahren neu aufscheinenden Herausforderungen, vor allem im Lichte der ökologischen Bedrohung, sahen sie die Notwendigkeit einer Neudefinition von Fortschritt. Ähnlich wie Amery ging es jedoch den wenigsten darum, Fortschritt per se zurückzuweisen. Ganz im Gegenteil: Das Austeritätsprogramm und die autoritären Staatsvorstellungen Herbert Gruhls und anderer konservativer Gründungsgrüner riefen heftige Kritik hervor. Für die Mehrheit der der jungen Partei war die Reformierung und Liberalisierung der westdeutschen Gesellschaft, die in den 1960er und 1970er Jahren stattgefunden hatte ${ }^{(54)}$, nicht verhandelbar. Darin trafen sie sich in den Positionen Amerys, der eine Rückbesinnung auf die geistighumanistischen Grundlagen von Fortschritt für erforderlich hielt.

\section{4. „Konservativismus“ und „Progressismus“, „rechts“ und „links“}

Die notwendige Voraussetzung für diese Rückbesinnung sei, so Amery, die Überwindung hergebrachter Kategorien und Lösungsmuster:

„Wer heute noch nostalgisch an den alten Parolen und Kategorien festhält [...], übersieht nicht nur die neuen Frontstellungen und die neuen Allianzen, - er übersieht die aktuelle Verpflichtung, die sich eben aus diesen alten Kategorien und aus dem alten, aber ewigjungen Engagement ergibt, dem Engagement der Aufklärung: die Verpflichtung nämlich, sich in jeder neu enthüllten Gefahr aufs neue des eigenen Verstandes zu bedienen “(55).

An erster Stelle sollte die Aufhebung der klassischen Trennung zwischen „konservativ“ und „progressiv“, „rechts“ und „links“ stehen, jener Kategorien also, die seit der Französischen Revolution die politisch-ideelle Landschaft Westeuropas strukturiert hatten ${ }^{(56)}$. Beiden Lagern sprach Amery angesichts der neu heraufziehenden Gefahren und Herausforderungen jegliche alleinige Lösungskompetenz ab:

„Es ist unverantwortlich so zu tun, als sei die Kernfrage dieses Jahres die Frage nach Freiheit oder Sozialismus. Unsere Enkel werden überhaupt nicht daran interessiert sein, ob sie aufgrund der Freiheit oder aufgrund des Sozialismus verheizt werden, für sie wird es um Brot, um Luft, um Wasser gehen - und um die anständigste Art, diese unabdingbaren Lebensgüter sicherzustellen. Weder soziale Marktwirtschaft noch Staatskapitalismus können das bewirken “(57).

Ein denkbar schlechtes Zeugnis stellte der bekennende Konservative zunächst dem bundesdeutschen Konservativismus aus. Zwar hätten sich, so Amery in einem Kommentar zur Chemiekatastrophe 1976 im italienischen Seveso, „sämtliche Einwände der konservativen Kulturkritik gegen das Industriesystem [...] bewahrheitet“, doch tauge die konservative Perspektive lediglich zum Erkennen, nicht jedoch zum

54 Zur These der Liberalisierung: Ulrich Her Bert (Hg.), Wandlungsprozesse in Westdeutschland. Belastung, Integration, Liberalisierung 1945-1980, Göttingen, Wallstein, 2002.

55 Carl Amery, „Der gefährliche Fortschritt. Zu einem Aufsatz von Jean Améry“, Frankfurter Hefte, 8 (1979), S. 15-18, hier: S. 18.

56 Vgl. dazu: Norberto Воввіо, Rechts und Links. Gründe und Bewertungen einer politischen Unterscheidung, Berlin, Wagenbach, 1994.

57 Carl Amery, Bausteine eines grünen Europa. [Ansprache] 1978 Marienplatz München (Monacensia, NL Carl Amery, Manuskripte: CA M 61 [Ms. 61]), S. 3. 
Bekämpfen der ökologischen Gefahren ${ }^{(58)}$. Der gegenwärtige Konservativismus sei tot oder allenfalls scheinlebendig, unfähig, sich auf die Neuen Sozialen Bewegungen und die Perspektive der politischen Ökologie einzulassen ${ }^{(59)}$. Diese weitgehende Sprachlosigkeit des Konservativismus gegenüber den neuen Problemlagen führte Amery auf zwei Ursachen zurück: Erstens auf sein Bündnis mit dem Nationalsozialismus, das einen weitgehend säkularen Konservativismus hervorgebracht habe, der die kulturelle Moderne zwar weiterhin ablehne, ihre technischen Segnungen jedoch in sein Programm integriert habe ${ }^{(60)}$; zweitens auf den Wandel des bundesdeutschen Konservativismus seit der Adenauerzeit, der sich dem Prinzip immerwährenden wirtschaftlichen Wachstums und damit dem rein materiellen Fortschritt verschrieben habe ${ }^{(61)}$. Dem Konservativismus der 1970er Jahre, parteipolitisch vertreten durch CDU und CSU, attestierte Amery einen „miserablen Stand“(62). Die Versuche der Union seit Mitte des Jahrzehnts unter den Stichworten „Tendenzwende“ und „geistig-moralische Wende“ die ideologische Lufthoheit der Republik wiederzugewinnen, sah Amery zum Scheitern verurteilt, da die wahren Herausforderungen der Zeit nicht erkannt würden ${ }^{(63)}$. Damit übte er Kritik an einem Konservativismus, der den klassischen konservativen Prinzipien Bewahrung und Tradition verstärkt marktliberale Werte vorzog. Solch ein Gestaltwandel des Konservativismus sollte sich in den 1980er Jahren am deutlichsten im Großbritannien Margaret Thatchers beobachten lassen ${ }^{(64)}$.

Doch die politische Linke kam in Amerys bissiger Analyse keinesfalls besser weg. „Nichts zeigt klarer den Wendecharakter, und zwar den Jahrtausendwendecharakter unseres Weltmoments an, als die Verwirrung des Marxismus durch die ökologische Krise“ ${ }^{(65)}$. Tatsächlich fielen die Diskussionen um den ökologischen Preis stetig steigenden Wirtschaftswachstums zeitlich und argumentativ mit der „Krise des Marxismus“ zusammen. Das Stillschweigen der Arbeiterklasse trotz steigender Arbeitslosigkeit und empfindlicher wirtschaftlicher Strukturkrisen seit Mitte der

58 Carl Amery, Kein Alibi für Seveso, Aug. 1976. Kulturkommentar (Monacensia, NL Carl Amery, Manuskripte: CA M 33 [Ms. 33]), S. 2f.

59 C. Amery, „Der gefährliche Fortschritt“ (Anm. 55), S. 18; Carl Amery, „Das Schicksal des Deutschen Konservativismus und die neuen sozialen Bewegungen“, in: C. Amery, Bileams Esel (Anm. 20), S. 30-49.

60 C. Amery, „Schicksal“ (Anm. 59), S. 43; Carl Amery, „Zivilisationskritik auf dem Abstellgleis“, in: Erhard O. Müller (Hg.), Weichen stellen zwischen Vision und Pragmatismus. Beiträge zum Generationendialog der Umweltbewegung. Dokumentation einer Veranstaltung der Heinich-Böll-Stiftung am 1. und 2. März 2001, Berlin 2001, S. 36-37, S. 7f.

61 C. Amery, „Schicksal“ (Anm. 59), S. 42; C. Amery, „Lebensqualität“ (Anm. 33), S. 11.

62 C. Amery, Ist ökologischer Humanismus möglich? (Anm. 13), S. 1.

63 Carl Amery, „Der konservative Selbstverrat. Gedanken zu einer ausgebliebenen ,Tendenzwende““, APuZ, 26/51 (1976), S. 3-5. Zu diesen Prozessen aus zeithistorischer Perspektive: Axel SCHILDT, „,Die Kräfte der Gegenreform sind auf breiter Front angetreten'. Zur konservativen Tendenzwende in den Siebzigerjahren“, Archiv für Sozialgeschichte, 44 (2004), S. 449-478; Andreas Wirsching, „Die mediale ,Konstruktion' der Politik und die ,Wende“ von 1982/83“, Historisch-Politische Mitteilungen, 9 (2002), S. 127-139.

64 Vgl. Dominik Geppert, Thatchers konservative Revolution. Der Richtungswandel der britischen Tories 1975-1979, München, Oldenbourg, 2002.

65 C. Amery, Natur als Politik (Anm. 43), S. 147. 
1970er Jahre, die Wirkungslosigkeit sozialistischer Rezepte in der spätkapitalistischen Gesellschaft und eben nicht zuletzt die Sprachlosigkeit linker Theorie im Angesicht der ökologischen Herausforderung führten seit Mitte der 1970er Jahre zu einer veritablen Identitätskrise der Linken überall in Westeuropa, die eng mit einer Krise des Fortschrittsbegriffs in diesen Reihen verbunden war ${ }^{(66)}$. In Westdeutschland fand die „Krise der Linken“ beredten Ausdruck in den Zerfallsprozessen der kommunistischen Kadergruppen oder den Suchbewegungen der undogmatischen Linken, die teilweise zu den Grünen führten ${ }^{(67)}$. Der Konservative Amery betrachtete diese Krise der Linken nicht ohne Häme: „Die Rezepte der Linken zu seiner, des Menschen, Befreiung müssen in solchem [ökologischen] Zusammenhang tatsächlich als abstrus erscheinen; als die Behauptung etwa, daß die Probleme eines untergehenden Passagierschiffs durch die Aufhebung der ersten und der zweiten Klasse gelöst werden könnten “ ${ }^{(68)}$. Im Angesicht der ökologischen Bedrohung gelte es nun den Gebrauch des sozialistischen Schlüsselbegriffs der „Ausbeutung“ auszuweiten. Er müsse nicht mehr bloß für die Beschreibung zwischenmenschlicher Herrschaftsverhältnisse unter dem Stichwort „Klassenkampf “ Verwendung finden, sondern sei zur Analyse des Verhältnisses von Mensch und Umwelt heranzuziehen ${ }^{(69)}$. Es komme darauf an, den Menschen endlich als Wesen zu begreifen, das in die Kreisläufe der Natur eingebunden sei und keinen autonomen Herrschaftsanspruch formulieren könne. An die Stelle des Historischen Materialismus müsse nun ein „ökologische[r] Materialismus“ treten: „Bisher hat sich der Materialismus begnügt, die Welt zu verändern; jetzt kommt es darauf an, sie zu erhalten“ ${ }^{(70)}$.

Eine Verschränkung konservativer und progressiver Elemente schien bereits bei der Analyse der neuartigen Problemlagen angebracht. Sprach Amery dem Konservativismus eine glaubhaftere Kritik der gesellschaftlichen und institutionellen Grundlagen der Wachstumsgesellschaft $\mathrm{zu}^{(71)}$, so sei wiederum der Sozialismus unentbehrlich, um die Herausforderungen zu meistern, die aus der Absage an das anthropozentrische Weltbild entstünden:

„Gefahren zur Inhumanität, die immer dann auftauchen, wenn man der (richtigen) Erkenntnis folgt, daß der Mensch nicht im Mittelpunkt steht. Die Panik, die aus solcher Erkenntnis zu entstehen vermag, bedarf jeder nur möglichen Korrektur durch die

66 Beispielhaft: Louis Althusser, Die Krise des Marxismus, Hamburg, VSA, 1978; André Gorz, Adieux au prolétariat, Paris, Galilée, 1980. Die „Krise der Linken“ in Frankreich fand u.a. auch ihren Ausdruck in der Entwicklung des Poststrukturalismus. Vgl. dazu auch das geschichtswissenschaftliche Dissertationsprojekt von Martin Kindtner (Trier/Tübingen), Propheten der Verflüssigung. Der französische Poststrukturalismus als Gegenwartsdiagnose und politisches Projekt.

67 Gerd Koenen, Das rote Jahrzehnt. Unsere kleine deutsche Kulturrevolution 1967-1977, Köln, Kiepenheuer \& Witsch, 2001; Andreas KüHN, Stalins Enkel, Maos Söhne. Die Lebenswelt der K-Gruppen in der Bundesrepublik der 70er Jahre, Frankfurt am Main u.a., Campus, 2005; S. Mende, „Nicht rechts, nicht links“(Anm. 1), S. 419-427.

68 Carl Amery, Definitionsschwierigkeiten des Konservatismus (Monacensia, NL Carl Amery, Manuskripte: CA M 100 [Ms. 100]), S. 4.

69 C. Amery, Progressismus, Konservatismus (Anm. 34), S. 8 f.

70 C. Amery, Natur als Politik (Anm. 43), S. 185.

71 C. Amery, „Der konservative Selbstverrat“ (Anm. 63), S. 3. 
humanistische Alternative. Und diese Alternative soll und muß auch vom methodischen Marxismus eingebracht werden [...]“(72).

Eine Verschränkung konservativer mit progressiven Elementen sei darüber hinaus bei der Suche nach Auswegen aus der planetarischen Bedrohung vonnöten. Das Leitmotiv gelte es aus dem Werk Edmund Burkes - in den Augen Amerys des "gescheitesten Konservativen überhaupt“ - zu entlehnen: Die „Partnerschaft der Toten, der Lebenden und der noch Ungeborenen“"(73). In der Wahl der Mittel jedoch biete der Konservativismus wenig Chancen: Mit konservativen Mitteln seien die konservativen Ziele nicht zu erreichen ${ }^{(74)}$. Stattdessen gelte es sich an linke Rezepte zu halten. Die Wende zum Besseren hin - Amery sprach wie viele andere Gründungsgrüne häufig von der Notwendigkeit einer „kopernikanischen Wende“ - könne allein durch eine Revolution des Bewusstseins herbeigeführt werden.

Die Grundbedingung zur Lösung der Probleme war nach Einschätzung Amerys also eine Überwindung des Dualismus von „konservativ“ und „progressiv“, eine Absage an den traditionellen Gegensatz von „rechts“ und „links“. Stattdessen gelte es, die Stärken beider Denkweisen miteinander zu kombinieren. Zur Überwindung namentlich der ökologischen Bedrohung solle auf dem Wege einer revolutionären Umwälzung des Bewusstseins das Prinzip der Bewahrung von Mensch und Schöpfung als handlungsleitend durchgesetzt werden. Diese Einschätzung teilte Amery mit einer Vielzahl von Gründungsgrünen. Den neuen Herausforderungen wollten sie mit neuen Begriffen, Kategorien und Lösungsmustern begegnen. Die Sprachlosigkeit eines stark auf wirtschaftliche Belange konzentrierten Konservativismus und der Bankrott sozialistischer Kategorien und Konzepte angesichts der neuen Problemlagen schien eine Überwindung des klassischen Gegensatzes von „rechts“ und „links“ zu erfordern, der - eng verknüpft mit dem Fortschrittsprinzip - die politisch-ideelle Landschaft Westeuropas seit der Französischen Revolution strukturiert hatte. Nur mithilfe einer solcherart gemeinsamen Anstrengung von „rechts“ bis „links“ - von „Dutschke bis Gruhl“, wie dieses Diktum seitens mancher Grüner personifizierend abgewandelt wurde - sei die gegenwärtige Krise zu meistern.

Die frühe grüne Wahlbewegung war beredter Ausdruck dieses auch von Amery forcierten Versuchs: Sie reichte von Antimodernisten mit völkischer Vergangenheit und bürgerlichen Naturschützern bis hin zu undogmatischen Sozialisten und Kommunisten. Die Präsenz unterschiedlich ausgeprägter Ideen eines „Dritten Weges“ innerhalb der frühen grünen Bewegung unterstreicht diese Suche nach dem Anderen und Neuen nochmals eindringlich. Das in der Frühzeit der Grünen häufig vorgetragene Mantra, sie seien „nicht rechts, nicht links, sondern vorn“, wurde zum Gründungskitt der Partei am Übergang zu den 1980er Jahren. In den ersten Jahren der Bewährung in den Niederungen von Tagespolitik und parlamentarischem Alltag zeigten sich jedoch schnell die Sollbruchstellen eines solchen Anspruches. Vor allem viele konservative

72 Carl Amery, „Die philosophischen Grundlagen und Konsequenzen der Alternativbewegung“, in: Hans-Werner Lüdke, Olaf Dinné (Hg.), Die Grünen. Personen - Projekte - Programme, Stuttgart, Seewald, 1980, S. 9-21, hier: S. 19.

73 C. Amery, Progressismus, Konservatismus (Anm. 34), S. 3f. Dieses konservative Grundmotiv scheint auch in dem Wahlslogan auf, mit dem die Grünen in den Bundestagswahlkampf 1980 zogen: „Wir haben die Erde von unseren Kindern nur geborgt.“ 
Grünen verließen rasch die noch junge Partei. Im Laufe der 1980er Jahre wurde diese zu einer linken Milieupartei, jedoch nicht ohne einige der Vorstellungen aus der Gründungsphase zu bewahren.

\title{
Zusammenfassung
}

Carl Amery steht für jenen Typus des grünen „Bewegungsintellektuellen“, der als regelrechte "Drehpunktperson“ zwischen der entstehenden grünen Bewegung und dem linksliberalen Milieu potentieller Unterstützer vermittelnd tätig wurde. Darüber hinaus forcierte er die Formierung der grünen Partei mit seinen weitreichenden Kontakten und Netzwerken und versuchte sie auf ein konsistentes theoretisches Fundament zu stellen, nicht zuletzt in einem über den Nationalstaat hinausgehenden, westeuropäischen Kontext. Seine Bedeutung für die frühen Grünen veranschaulicht, wie sehr eine soziale Bewegung, die sich zunächst weitgehend jenseits der „Mehrheitsgesellschaft" formierte, solch intermediärer Persönlichkeiten bedurfte. Sie vermittelten zwischen den Sphären und verliehen der Bewegung über ihre eigene Anhängerschaft hinaus Unterstützung und Glaubwürdigkeit.

\begin{abstract}
New Social Movements that emerged all over Western Europe in the 1970s held a deep-rooted skepticism towards any kind of outstanding protagonists, leaders or spokespersons in their own rows. So did the green movement in West-Germany as well as the early green party founded in 1980. What we can see in this period is nevertheless the emergence of some charismatic figures who played more important roles than others within the party organization. This article focuses on one of them, the Bavarian author and green activist Carl Amery, and intends to ask if Amery could be described as kind of a green "movement intellectual". Starting with the correspondent concept outlined by social scientists Ron Eyerman and Andrew Jamison and followed by a brief biographical sketch of Carl Amery, the analysis concentrates on his ideas of progress and conservatism as well as his attitudes towards the traditional left-right-dichotomy - both key questions at the centre of political discussion in the West German green movement and later in the early green party.
\end{abstract}

\section{Résumé}

Les nouveaux mouvements sociaux émergeant partout en Europe de l'Ouest dans les années 1970 cultivaient un grand scepticisme à l'égard des protagonistes prédominants dans leurs propres rangs. Le mouvement vert en RFA ainsi que le parti des Verts fondé en 1980 en faisaient autant. Pourtant, déjà à l'époque, dans l'organisation et le quotidien du Parti, certains personnages jouaient un rôle plus important que d'autres. Cet article se focalise sur l'un d'eux, l'auteur bavarois et l'activiste écologique Carl Amery. Peut-il être qualifié de véritable " movement intellectual " parmi les premiers Verts? Commençant avec le concept correspondant, développé par Ron Eyerman et Andrew Jamison, et suivi par un bref aperçu biographique d'Amery, l'analyse se concentre sur sa pensée concernant le conservatisme et le progrès, ainsi que sur ses positions face à la dichotomie traditionnelle droite-gauche. Cela nous mène au cour des questions politiques débattues avec ferveur parmi les Verts dans leur phase de formation. 